\title{
Avaliação da qualidade de vida dos pacientes em uso de fixador externo circular em um hospital de trauma*
}

\section{Evaluation of the Quality of Life of Patients Using Circular External Fixator in a Trauma Hospital}

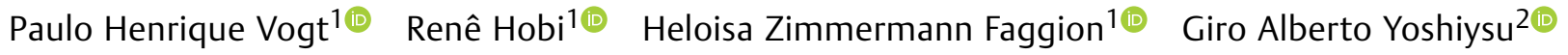 \\ Lucas Antônio Ferraz Marcon ${ }^{1}$ Cássio Kenji Hiraga ${ }^{10}$
}

${ }^{1}$ Departamento de Ortopedia e Traumatologia, Hospital do Trabalhador, Universidade Federal do Paraná, Curitiba, PR, Brasil

2 Departamento de Residência Médica, Hospital do Trabalhador, Universidade Federal do Paraná, Curitiba, PR, Brasil, Curitiba, PR, Brasil

Rev Bras Ortop 2022;57(2):214-217.

\begin{abstract}
Endereço para correspondência Paulo Henrique Vogt, MD, Av. Rep. Argentina, 4406 - Novo Mundo, Curitiba, PR, CEP: 81050-000, Brasil (e-mail: paulovogt90@gmail.com).
\end{abstract}

\begin{abstract}
Resumo
Objetivo Avaliar a qualidade de vida dos pacientes em uso do fixador externo do tipo Ilizarov para tratamento de fraturas complexas e de suas sequelas

Método Trata-se de um estudo observacional e transversal, em que foi aplicado o questionário 36-item short form survey (SF-36) (traduzido para a língua portuguesa) durante as consultas ambulatoriais em 2 períodos, nos meses de julho de 2018 e janeiro de 2019. Os pacientes que participaram do estudo realizaram seus procedimentos cirúrgicos no período de janeiro a junho de 2018.

Resultados Foram avaliados 36 pacientes em uso de fixador externo do tipo llizarov. Foi observado predomínio do sexo masculino e idade média de 37,9 anos. As fraturas

Palavras-chave

- qualidade de vida

- técnica de llizarov

- fixadores externos

- alongamento ósseo

- fraturas ósseas

- pseudoartrose de ossos da perna e suas complicações representaram metade da amostra. Foi observada melhora na capacidade funcional e nos aspectos emocionais dos pacientes ao longo do tratamento.

Conclusão O uso do fixador externo circular constitui um método importante e eficaz para o tratamento cirúrgico de fraturas complexas e de suas sequelas. Este trabalho permitiu concluir que, após o tratamento, os pacientes alcançaram retorno funcional às atividades diárias com adequada qualidade de vida.
\end{abstract}

Trabalho desenvolvido no Serviço de Ortopedia e Traumatologia do Hospital do Trabalhador, Curitiba, PR, Brasil. recebido

10 de Agosto de 2020

aceito

08 de Março de 2021

Publicado on-line

Outubro 13, 2021
DOI https://doi.org/

$10.1055 / \mathrm{s}-0041-1732383$

ISSN 0102-3616.

\footnotetext{
(c) 2021. Sociedade Brasileira de Ortopedia e Traumatologia. All rights reserved.

This is an open access article published by Thieme under the terms of the Creative Commons Attribution-NonDerivative-NonCommercial-License, permitting copying and reproduction so long as the original work is given appropriate credit. Contents may not be used for commercial purposes, or adapted, remixed, transformed or built upon. (https://creativecommons.org/ licenses/by-nc-nd/4.0/)

Thieme Revinter Publicações Ltda., Rua do Matoso 170, Rio de Janeiro, RJ, CEP 20270-135, Brazil
} 
Abstract

\section{Keywords}

- quality of life

- Ilizarov technique

- external fixators

- bone lengthening

- fractures, bones

- pseudoarthrosis
Objective To evaluate the quality of life of patients using an llizarov type external fixator for the treatment of complicated fractures and their sequelae.

Method This is an observational and cross-sectional study, in which the 36-item short form survey (SF-36) questionnaire (translated into Portuguese) was applied during outpatient consultations in 2 periods, in the months of July 2018 and January 2019. The patients who participated in the study underwent their surgical procedures between January and June 2018.

Results We evaluated 36 patients using an external Ilizarov fixator. We observed a predominance of male patients, with a mean age of 37.9 years. Fractures of leg bones and their complications represented half of the sample. Improvement in functional capacity and emotional aspects of the patients was observed throughout the treatment.

Conclusion The use of the circular external fixator is an important and effective method for the surgical treatment of complex fractures and their sequelae. This study allowed us to conclude that, after treatment, patients achieved functional return to daily activities with adequate quality of life.

\section{Introdução}

O uso de fixador externo como opção terapêutica no tratamento de fraturas ou alongamento ósseo foi incorporado às práticas da ortopedia em meados do século XIX. Por meio de muitos estudos, Codivilla, em Bolonha (Itália), realizou o primeiro estudo de alongamento ósseo, com o uso de aparelhos gessados, ${ }^{1}$ demonstrando a eficácia e as possibilidades que este método poderia proporcionar. ${ }^{1} \mathrm{Na}$ década de 1950, Gavrill Abramovich Ilizarov desenvolveu o fixador externo circular que levou seu nome, assim como o método de Ilizarov, baseado no conceito de osteogênese por distração. ${ }^{2,3}$ A distração osteogênica permite que o cirurgião realize o alongamento do membro em pacientes com discrepância de comprimento pós-traumática ocasionada pelo trauma ósseo em si ou secundário ao desbridamento da osteomielite. ${ }^{4}$ Ilizarov empregou nos seus estudos a taxa de distração segura para facilitar a formação óssea de $1 \mathrm{~mm}$ por dia. ${ }^{4,5}$ Devido a isto, é importante destacar que o período de tratamento com o fixador costuma ser longo, cerca de 15 meses nos casos de alongamento ósseo, ${ }^{4}$ resultando em alterações, mesmo que transitórias, no cotidiano, qualidade de vida e funcionalidade dos pacientes. Para Baschera et al., ${ }^{6}$ o tratamento com o fixador externo circular é eficaz e cumpre sua função biológica para o tratamento proposto. Porém, nota-se que o retorno às atividades laborais prévias não ocorreu no mesmo intervalo de tempo para todos os pacientes envolvidos. Já para Elsoe et al., ${ }^{7}$ após o tratamento com o fixador externo circular, as taxas de depressão moderada a severa se elevaram. Os autores associam esse dado ao grau complexo das fraturas apresentadas, ao tempo de tratamento e às consequências e limitações socioeconômicas que o longo seguimento terapêutico impõe. Conforme os cuidados exigidos durante a terapêutica, o presente estudo visa avaliar, em um hospital público terciário nível 1 em trauma, as repercussões geradas pelo método de Ilizarov na qualidade de vida (funcional e psicossocial) dos pacientes em tratamento com o fixador externo circular.

\section{Material e método}

Este estudo possui delineamento observacional transversal e avalia a qualidade de vida (física e psicossocial) dos pacientes em tratamento com fixador externo circular (método de Ilizarov) para tratamento das complicações decorrentes de fraturas e lesões complexas em membros inferiores. A coleta de dados foi realizada em 2 períodos, em julho de 2018 e janeiro de 2019. Os critérios de inclusão utilizados foram os pacientes que faziam uso do fixador externo circular em membros inferiores durante a aplicação do questionário e que concordaram em participar do estudo, assinando o termo de consentimento livre e esclarecido (TCLE). Foram excluídos os pacientes submetidos a fixadores externos com montagens não padrão e pacientes com prejuízo cognitivo ou distúrbios psiquiátricos severos que comprometessem a aplicação do questionário. Para a coleta de dados foi utilizado o questionário 36-item short form survey (SF-36) traduzido, adaptado e validado para a língua portuguesa. ${ }^{8}$ Este é um instrumento genérico de avaliação de qualidade de vida, de fácil aplicação e compreensão, formado por 36 itens em 8 grupos: capacidade funcional, aspectos físicos, dor, estado geral da saúde, vitalidade, aspectos sociais, aspectos emocionais e de saúde mental. ${ }^{8}$

Foram avaliados 36 pacientes no presente estudo, que responderam ao questionário em dois períodos distintos, mantendo-se 6 meses de diferença entre a primeira aplicação e a segunda. O período do tratamento no qual foi realizado o questionário variou amplamente entre os pacientes, com uma média de 11 meses. Do total avaliado, $75 \%$ eram do sexo masculino e $25 \%$ do sexo feminino. A faixa etária variou entre 19 e 63 anos de idade, sendo a média de 37,9 anos, e a mediana 38 anos. Em cerca de 80\% dos pacientes avaliados no estudo, o uso do fixador circular 
externo foi indicado para o tratamento de complicações de fraturas de ossos da perna, sendo elas a pseudoartrose (PSA), osteomielite, falha óssea e/ou consolidação viciosa. No período avaliado, desde o trauma inicial, os pacientes foram submetidos a múltiplos procedimentos cirúrgicos antes da montagem do sistema de Ilizarov; em média, cada paciente foi submetido a 4 procedimentos que envolviam estabilização cirúrgica, desbridamentos de tecidos moles e/ou ressecção óssea, sendo que a média de permanência do método para o término do tratamento proposto ocorreu em torno de 15,6 meses.

Para a análise dos dados, foi utilizado o programa estatístico R Versão 3.5.1 (R Core Team, Viena, Áustria), software livre e de código aberto. A análise descritiva foi realizada verificando as quantidades e percentuais para as variáveis categóricas e medidas descritivas (mínimo, máximo, quartis, média e desvio padrão) para as variáveis contínuas.

\section{Resultados}

Dentre os itens avaliados (capacidade funcional, limitação por aspectos físicos, dor, estado geral de saúde, vitalidade e aspectos sociais) através do questionário SF-36, apenas capacidade funcional e aspectos emocionais do paciente revelaram alterações estatisticamente significativas.

Constatou-se por meio da aplicação do questionário e com uso do teste $\mathrm{t}$ pareado que a capacidade funcional dos pacientes aumentou em média 3,71 pontos $(p<0,05)$ quando comparada ao estado funcional prévio traumático ou infeccioso, com um $p<0,001$ entre os parâmetros avaliados. (- Tabela 1) (valores iniciais 30,32 $\pm 24,66$ e valores finais $34,03 \pm 23,47$ ). Esse valor demonstrou diferença significativa e indicou a melhora do paciente com o tratamento, da mesma forma, por análise deste subitem, é possível afirmar que os pacientes conseguem, é possível afirmar que os pacientes conseguem, mesmo em uso do fixador externo, retornar a exercer as suas atividades habituais e básicas do dia a dia, como tomar banho sem assistência, andar nas ruas, utilizar o transporte público, fazer compras em mercados.

Tabela 1 Comparativo pareado entre antes e depois para as dimensões com valores de $p<0,05$

\begin{tabular}{|l|l|l|l|}
\hline Quesitos avaliados & Média & DP & $\begin{array}{l}\text { Valor de } \\
\mathbf{p}<\mathbf{0 , 0 5}\end{array}$ \\
\hline Capacidade funcional & & & $\mathbf{0 , 0 0 1}$ \\
\hline Antes & 30,32 & 24,66 & \\
\hline Depois & 34,03 & 23,47 & \\
\hline Diferença & 3,71 & 4,28 & \\
\hline Aspectos emocionais & & & $\mathbf{0 , 0 3}$ \\
\hline Antes & 25,81 & 33,01 & \\
\hline Depois & 34,41 & 42,59 & \\
\hline Diferença & 8,6 & 21,03 & \\
\hline
\end{tabular}

Abreviaturas: DP, desvio padrão.
O aspecto emocional foi, também, outro subitem do questionário que apresentou alterações significativas. Neste ponto, observa-se, inclusive, que houve um aumento de 8,6 pontos $(p<0,05)$ ( - Tabela 1$)$ entre a primeira aplicação do questionário e a segunda, com uma diferença de 6 meses entre elas, com um $p<0,03$ entre os parâmetros avaliados.

E ainda, 70,5\% dos pacientes acreditam que, comparativamente, sua percepção de saúde no momento da aplicação do questionário era melhor do que no início do tratamento com o método, a percepção da sua saúde obteve melhora ou manutenção em níveis aceitáveis. Pode-se avaliar que, durante o tratamento e a sua progressão, os pacientes relataram estar mais estimulados e, em consequência, mais envolvidos com o tratamento e recuperação.

\section{Discussão}

Neste heterogêneo grupo de pacientes submetidos ao método do fixador externo circular por complicações traumáticas, como pseudoartrose ou infecção óssea, 70,5\% acreditam que sua saúde melhorou quando comparada ao período prévio ao início do tratamento. A melhora da capacidade funcional e dos aspectos emocionais demostrada pelo questionário SF-36 pode estar relacionada com a estabilização do quadro clínico inicial, com o entendimento pelo paciente da complexidade da sua lesão ou com o uso e os resultados obtidos pelo do método de Ilizarov, como demonstrado por Wang et al. ${ }^{9}$

Porém, a melhora do quadro funcional pode estar relacionada ao objetivo do fixador externo circular, que é o tratamento das pseudoartroses, osteomielites e fraturas complexas. Sendo assim, a evolução do quadro do paciente e o retorno gradual às suas atividades demonstram melhora funcional do quadro prévio. $\mathrm{O}$ retorno às atividades, e a evolução positiva do quadro com bons resultados do método Ilizarov podem estar relacionados com a melhora do aspecto emocional do paciente.

A satisfação com o uso do fixador externo circular obtida em nosso estudo, demostrada pela melhora emocional dos pacientes, está de acordo com a literatura, como demostrado por Baschera et al. ${ }^{6}$ em trabalho publicado em 2014. Por meio do questionário SF-12, foi constatado que o índice de satisfação da amostra estudada com o tratamento com fixador externo circular (método de Ilizarov) foi de 96,8\%. Neste mesmo estudo, $91,7 \%$ dos pacientes responderam que se submeteriam ao mesmo tratamento novamente dentro de circunstâncias similares caso fosse necessário.

Wang et al., ${ }^{9}$ em 2017, também avaliaram o componente mental pelo SF-36 em sua série de pacientes. Notaram melhora do estado psicológico conforme a progressão do tratamento (os valores encontrados foram de $37,5 \pm 4,5$ e ao final do tratamento, chegaram a 72,5 $\pm 5,5 ; \operatorname{com} p=0,00)$. Em relação ao fator psicológico e emocional, foi possível afirmar com este estudo que os pacientes em uso de fixador externo circular tiveram uma melhora nos sinais de ansiedade ou depressão.

Em publicação de 1997, McKee et al. ${ }^{10}$ avaliaram 25 pacientes através do SF-36, com aplicação do questionário no pré- e no pós-operatório. No escore geral, assim como o 
presente estudo, foram revelados valores que representam melhora da atividade funcional. Porém, assim como observado neste presente estudo, quesitos como vitalidade ( $37 \pm 4$ para $46 \pm 7$ ) e função física ( $38 \pm 6$ para $46 \pm 7$ ) não apresentaram melhora significativa, com índices fora dos intervalos de confiabilidade.

Como trata-se de pacientes com complicações ortopédicas e paciente decorrentes de fraturas, a avaliação funcional e emocional pré-operatória torna-se complicada; porém, a literatura e nosso trabalho estão em consonância em relação aos bons resultados do método de Ilizarov e à adequação/adaptação do paciente ao método.

\section{Conclusão}

Apesar da grande complexidade das fraturas apresentadas, complicações, tempo de tratamento e das consequências e limitações socioeconômicas que o seguimento terapêutico impõe, os pacientes elencados para uso do método do fixador externo circular de Ilizarov obtiveram melhora funcional e dos aspectos emocionais em relação ao início do tratamento.

\section{Suporte Financeiro}

Não houve suporte financeiro de fontes públicas, comerciais, ou sem fins lucrativos.

Conflito de interesses

Os autores declaram não haver conflito de interesses.

\section{Referencias}

1 Codivilla A. The classic: On the means of lengthening, in the lower limbs, the muscles and tissues which are shortened through deformity. 1905. Clin Orthop Relat Res 2008;466(12):2903-2909

2 Vargas Barreto B, Caton J, Merabet Z, Panisset JC, Pracros JP. Complications of Ilizarov leg lengthening: a comparative study between patients with leg length discrepancy and short stature. Int Orthop 2007;31(05):587-591

3 Brinker MR, O'Connor DP. Outcomes of tibial nonunion in older adults following treatment using the Ilizarov method. J Orthop Trauma 2007;21(09):634-642

4 Schep NW, van Lieshout EM, Patka P, Vogels LM. Long-term functional and quality of live assessment following post-traumatic distraction osteogenesis of the lower limb. Strateg Trauma Limb Reconstr 2009;4(03):107-112

5 Aronson J. Limb-lengthening, skeletal reconstruction, and bone transport with the Ilizarov method. J Bone Joint Surg Am 1997;79 (08):1243-1258

6 Baschera D, Kingwell D, Wren M, Zellweger R. A holistic perspective of patients' lives post-Ilizarov external fixation. ANZ J Surg 2014;84(10):776-780

7 Elsoe R, Kold S, Larsen P, Petruskevicius J. A prospective observational study of 56 patients treated with ring fixator after a complex tibial fracture. Strateg Trauma Limb Reconstr 2017;12(01):35-44

8 Ciconeli RM, Ferraz MB, Santos W, Meinão I, Quaresma MR. Tradução para a língua portuguesa e validação do questionário genérico de avaliação de qualidade de vida SF-36 (Brasil SF-36). Rev Bras Reumatol 1999;39(03):143-150

9 Wang H, Wei X, Liu P, et al. Quality of life and complications at the different stages of bone transport for treatment infected nonunion of the tibia. Medicine (Baltimore) 2017;96(45):e8569

10 McKee MD, Yoo D, Schemitsch EH. Health status after Ilizarov reconstruction of post-traumatic lower-limb deformity. J Bone Joint Surg Br 1998;80(02):360-364 